EPJ manuscript No.

(will be inserted by the editor)

\title{
Deconfinement and Quarkonium Suppression
}

\author{
Frithjof $\operatorname{Karsch}^{1,2}$ a \\ 1 Fakultät für Physik, Universität Bielefeld, D-33615 Bielefeld, Germany \\ 2 Physics Department, Brookhaven National Laboratory, Upton, NY 11973, USA \\ Received: date / Revised version: date
}

\begin{abstract}
Modifications in the production pattern of heavy quark bound states have long been considered to provide sensitive signatures for the thermal properties of dense matter created in heavy ion collisions. The original concept of Matsui and Satz for quarkonium suppression as signature for deconfinement in heavy ion collisions has been challenged recently through lattice studies of spectral functions, which indicate the persistence of heavy quark bound states at temperatures well above the transition, as well as through the refined analysis of hadronization and recombination models, which take into account the thermal evolution of the medium generated in a heavy ion collision. We will review here recent developments on these topics.
\end{abstract}

PACS. 11.15.Ha , 11.10.Wx, 12.38.Mh, 25.75.Nq

\section{Introduction}

The concept of quarkonium suppression as a probe for the thermal properties of hot and dense particle matter or, more specifically, for the deconfining nature of the transition between hadronic matter at low temperatures and a dense partonic medium at high temperatures has originally been put forward by Matsui and Satz 1. In their approach they utilized the long-distance infrared properties of QCD; in a deconfined medium the heavy quark ' potential gets screened and at sufficiently high tempera- ture the screening radius will be smaller than the typical size of a quarkonium state. As a consequence the screened potential no longer can support the formation of bound states.

Screening strongly depends on a sufficiently high density of partons, i.e. color charges that can contribute to the screening. In a QED plasma, for instance, the screening radius $r_{D}$ and density $n$ are related through $r_{D} \sim 1 / g \sqrt{n / T}$. The rapid rise of the parton density at a well defined transition temperature, irrespective of this being a phase transition temperature or a temperature characterizing a rapid crossover, thus intimately connects the occurrence of a (phase) transition in QCD with the rapid decrease of the screening radius and thus also with the fate of quarkonium bound states.

These qualitative considerations, however, illustrate already the difficulty that arises when one wants to use the concept of quarkonium suppression as a quantitative tool for studies of the QCD transition. On the one hand, it is the long distance property of QCD which undoubtedly

\footnotetext{
a Present address: Physics Department, Brookhaven National Laboratory, Upton, NY 11973, USA
}

will prohibit the formation of heavy quark bound states at high temperature. On the other hand, as heavy quark bound states are small in size also on the typical scale of QCD, $1 / \Lambda_{Q C D} \sim 1 \mathrm{fm}$, screening has to be strong enough to modify also the short distance part of the QCD potential. Already in the simple context of equilibrium thermodynamics different length scales thus play a role which have to be understood quantitatively before quarkonium suppression can be established as a unique signature for the existence of a phase transition or at least for the characterization of the properties of the hot and dense high temperature phase; a difficulty which is common to many signatures for the QCD transition that are currently discussed.

A second ingredient to the concept of quarkonium suppression in heavy ion collisions which also has been utilized in [1] is that the creation of a heavy quark pair in these collisions is a rare event; it is assumed that a $q \bar{q}$-pair that is created in a dense medium and thus cannot form a bound state will separate unhampered and thereafter both quarks will no more find another heavy quark as partner to form a quarkonium bound state during the stage of hadronization. While this was a good approximation for the kinematic conditions at the SPS it might not be appropriate anymore at RHIC or LHC. Consequently it recently has been suggested that recombination processes [2] during the cooling of a hot plasma and subsequent pairing of heavy quarks initially originating from different creation processes (statistical hadronization 4,5]) may lead to a different suppression pattern or even to enhanced yields of heavy quark bound states.

A quantitative understanding of the heavy quark potential at finite temperature, the relevant lengths scales that characterize its short and long distance properties 

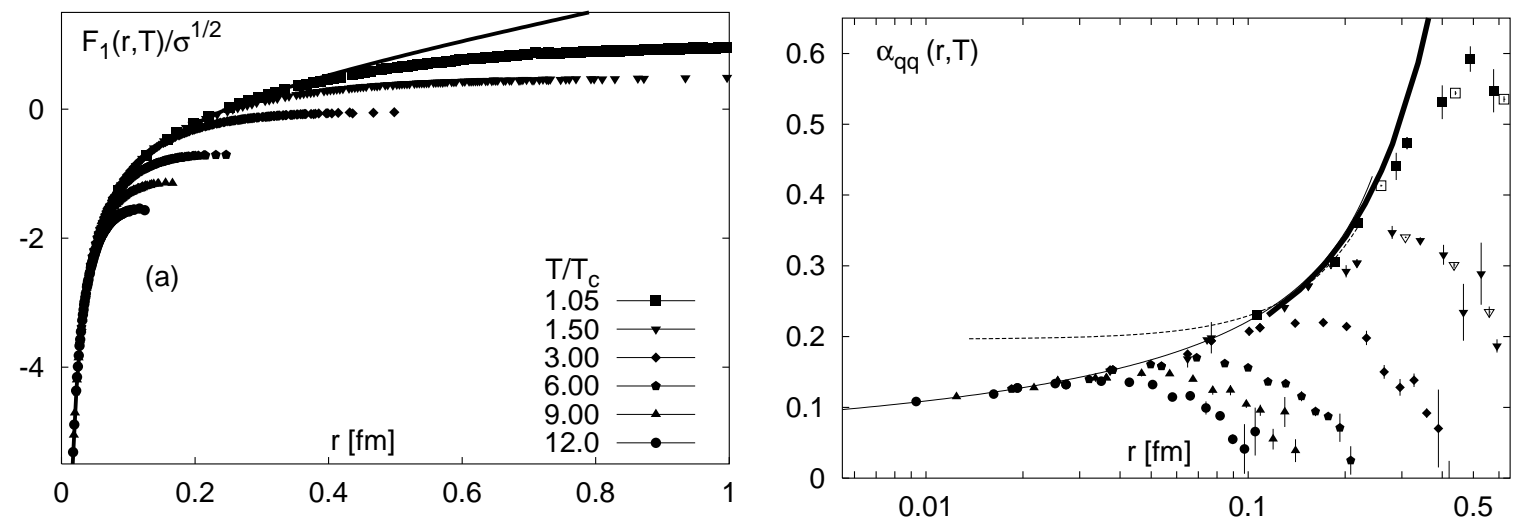

Fig. 1. Singlet heavy quark free energies (left) and the running coupling in the so-called $q q$-scheme (right) for temperatures above the transition temperature $T_{c}$. Results shown are obtained from calculations in pure SU(3) gauge theory [13].

and the in-medium properties of bound states combined with a thorough analysis of the time evolution of dense matter created in heavy ion collisions thus is needed to convert experimentally measured heavy quarkonium yields into a theoretically well understood tool that can be used for the detailed tomography ${ }^{1}$ of hot and dense matter. In fact, right from the beginning this was understood and has led to the discussion of sequential suppression patterns for bound states with different quantum numbers [6, its momentum and size dependence [7] as well as the role of competing suppression mechanisms in ordinary hadronic matter [8]. All this has been discussed in detail in a recent report on the future heavy ion program at the LHC 9].

We will concentrate here on a discussion of recent lattice calculations which aim at a better understanding of quarkonium properties at finite temperature. Through the detailed study of short, medium and long-distance properties of heavy quark free energies on the one hand and through calculations of spectral functions from thermal quarkonium correlation functions on the other hand much better inside into the fate of quarkonium states at high temperature has been obtained. In particular, a consistent picture now seems to emerge from potential model calculations, based on calculations of heavy quark free energies, and the direct spectral analysis of thermal hadron correlation functions.

We will start in the next section by discussing information on lengths scales relevant for the analysis of quarkonium bound states. Section 3 contains a discussion of the calculation of effective potentials from heavy quark free energies and their role in potential models. In Section 4 we present results on quarkonium spectral functions obtained through a maximum entropy analysis of thermal hadron correlation functions. A brief discussion of the consequences of these results for the analysis of quarkonium yields in heavy ion collisions is given in Section 5. Finally we give our Conclusions in Section 6 .

\footnotetext{
${ }^{1}$ to use a modern buzzword in heavy ion phenomenology
}

\section{Short and long distance scales at finite temperature}

A quite common qualitative argument for the dissolution of heavy quark bound states is that these can no longer exist in a hot and dense medium once the screening radius, i.e. the inverse of the (Debye) screening mass $r_{D} \equiv 1 / m_{D}$, becomes smaller than the typical size of a quarkonium bound state. To go beyond such qualitative arguments it is necessary to analyze more quantitatively what small and large distances mean in quarkonium physics at a given temperature. Some insight into this question can be gained by analyzing the short distance properties of excess free energies of a static quark anti-quark pair in a thermal medium [10]

$$
\begin{aligned}
\mathrm{e}^{-F_{\mathrm{av}}(r, T)} & =\frac{Z_{\bar{q} q}(T, V)}{Z(T, V)} \\
& \equiv \frac{1}{V} \sum_{\boldsymbol{x}, \boldsymbol{y} ;|\boldsymbol{x}-\boldsymbol{y}|=r} \frac{1}{9}\left\langle\operatorname{Tr} L(\boldsymbol{x}) \operatorname{Tr} L^{\dagger}(\boldsymbol{y})\right\rangle
\end{aligned}
$$

or the related singlet free energy, which is obtained from static quark anti-quark sources with fixed color orientation corresponding to a color singlet state,

$$
\mathrm{e}^{-F_{1}(r, T)}=\frac{1}{V} \sum_{\boldsymbol{x}, \boldsymbol{y} ;|\boldsymbol{x}-\boldsymbol{y}|=r} \frac{1}{3}\left\langle\operatorname{Tr} L(\boldsymbol{x}) L^{\dagger}(\boldsymbol{y})\right\rangle_{g f} .
$$

Here $L(\boldsymbol{x}$ denotes the Polyakov loop constructed in lattice calculations from a product of $\mathrm{SU}(3)$ matrices that represent the gluon fields [11. In Eq. 2 the subscript, $g f$, indicates that the expectation value has to be evaluated in a fixed gauge as the operator used to define $F_{1}$ is not gauge invariant. The corresponding gauge invariant operator, for instance in Coulomb gauge, would be a complicated non local object 12 .

The singlet free energy calculated in Coulomb gauge is shown in Fig. 1(left) for a selected set of temperatures above the transition temperature. The results shown are obtained from a calculation in a pure $\mathrm{SU}(3)$ gauge theory 
13. Recent results obtained in 2-flavor [14 and 3-flavor [15] QCD are, however, also on a quantitative level quite similar. As can be seen, $F_{1}(r, T)$ coincides with the zero temperature heavy quark potential,

$$
V(r)=-\frac{4}{3} \frac{\alpha}{r}+\sigma r
$$

for a certain range of quark anti-quark separations and then turns over quite rapidly into a constant that decreases with temperature. In fact, these features become much more transparent from a calculation of the running coupling as a function of distance and temperature 13 . 16. It can conveniently be defined from a derivative of the singlet free energy ( $T=0$ : force) which eliminates otherwise uncontrolled constants,

$$
\alpha_{\mathrm{qq}}(r, T)=\frac{3 r^{2}}{4} \frac{\mathrm{d} F_{1}(r, T)}{\mathrm{d} r} .
$$

This is shown in Fig. 1(right). At zero temperature the Cornell type potential, Eq. 3. yields $\alpha_{\mathrm{qq}}(r, 0)=\alpha+3 \sigma r^{2} / 4$, with $\alpha=\pi / 16$ arising in string models as correction to the linear rising string tension term. This is represented in Fig. 1 by the dashed line at short distances and the fat solid line at large distances. Within this parametrization of the potential the entire $r$-dependence of the coupling arises from the long distance confinement part of the potential. Replacing the constant coupling in the Cornell potential by the perturbatively running QCD coupling leads to a characteristic $r$-dependence also at short distances which is indicated by the thin solid line Fig. 11 It generally is argued that at $T=0$ the perturbative calculation of the coupling in the so-called $q q$-scheme (Eq. 4) is under control for $r \lesssim 0.1 \mathrm{fm} \mathrm{[16.17.}$

At short distances the running coupling at finite temperature coincides with the $T=0$ coupling and, of course, runs with the dominant scale given by the quark antiquark separation,

$$
\alpha_{\mathrm{qq}}(r, T) \sim \frac{6 \pi}{\left(33-2 N_{f}\right) \ln (1 / r \Lambda)}, \text { for } r \Lambda \ll 1 .
$$

At large distances, however, the coupling defined through Eq. [4 drops exponentially,

$$
\alpha_{\mathrm{qq}}(r, T) \sim \alpha(T) \exp \left\{-m_{D}(T) r\right\}, \text { for } r \gg 1 / T,
$$

which is in agreement with high temperature perturbation theory in a regime where temperature is the dominant scale.

Similar results as those shown in Fig. 1(left) for the running coupling of the pure gauge theory have been obtained also in 2 and 3 flavor QCD [14,15]18. They clearly show that (i) the coupling stays temperature independent in the short distance perturbative regime for quite a wide range of temperatures above $T_{c}$ and (ii) it does so also in the non-perturbative regime for temperatures up to about $1.5 T_{c}$; i.e. the coupling $\alpha_{\mathrm{qq}}(r, T)$ continues to show remnants of the confining force up to these temperatures. Moreover, (iii) the transition to the expected large distance behavior, Eq. [6] proceeds quite rapidly at a well defined distance, $r_{\max }$, which can be attributed to the point

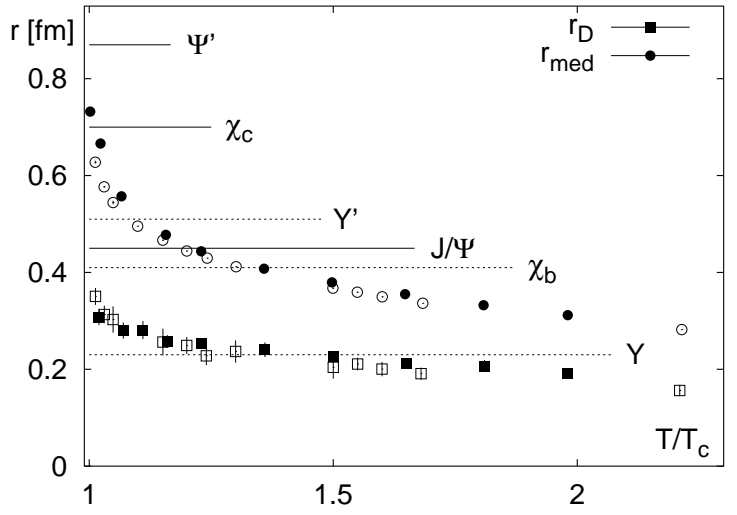

Fig. 2. The scale $r_{m e d}$ which gives an estimate for the distance beyond which the force between a static quark anti-quark pair is strongly modified by temperature effects and the Debye screening radius, $r_{D} \equiv 1 / m_{D}$. Open (closed) symbols correspond to $\mathrm{SU}(3)$ (2-flavor QCD) calculations. The horizontal lines give the mean squared charge radii of some charmonium and bottomonium states.

where $\alpha_{q q}(r, T)$ reaches a maximum at fixed $T$. Due to the rapid crossover from short to large distance behavior above $T_{c}$ this length scale is, in fact, well be approximated by the scale $r_{\text {med }}$ [14,19] defined as the point at which $F_{\infty} \equiv \lim _{r \rightarrow \infty} F_{1}(r, T)$ equals the zero temperature potential $V(r)$. This scale, extracted in a pure gauge theory [19] and 2-flavor QCD [14, is shown in Fig 2 together with the scale set by the inverse Debye mass, $r_{D} \equiv 1 / m_{D}$, which is extracted from the long-distance exponential screening of the singlet free energy. Also included in this figure as horizontal lines are mean squared charge radii of some charmonium (solid) and bottomonium (dashed) states which characterize the average separation $r$ entering the effective potential in the Schrödinger equation. It is reasonable to expect that the temperatures at which these radii equal $r_{\text {med }}$ provides a first estimate for the onset of thermal effects in quarkonium states. Of course, as the wave functions of the various quarkonium states do also reach out to larger distances [20] this can only be taken as a first indication for the relevant temperatures. The analysis of bound states using, for instance, the Schrödinger equation will do better in this respect.

\section{Non-Relativistic Bound State Models and the Heavy Quark Free Energies}

The influence of a thermal medium on heavy quark bound states is commonly discussed in terms of a non-relativistic, effective Hamiltonian for the quark anti-quark system [6] 21,22. Here the influence of the thermal medium is expressed in terms of a temperature dependent potential for the two-quark system which then can be used to study the bound state problem, for instance, by solving the cor- 

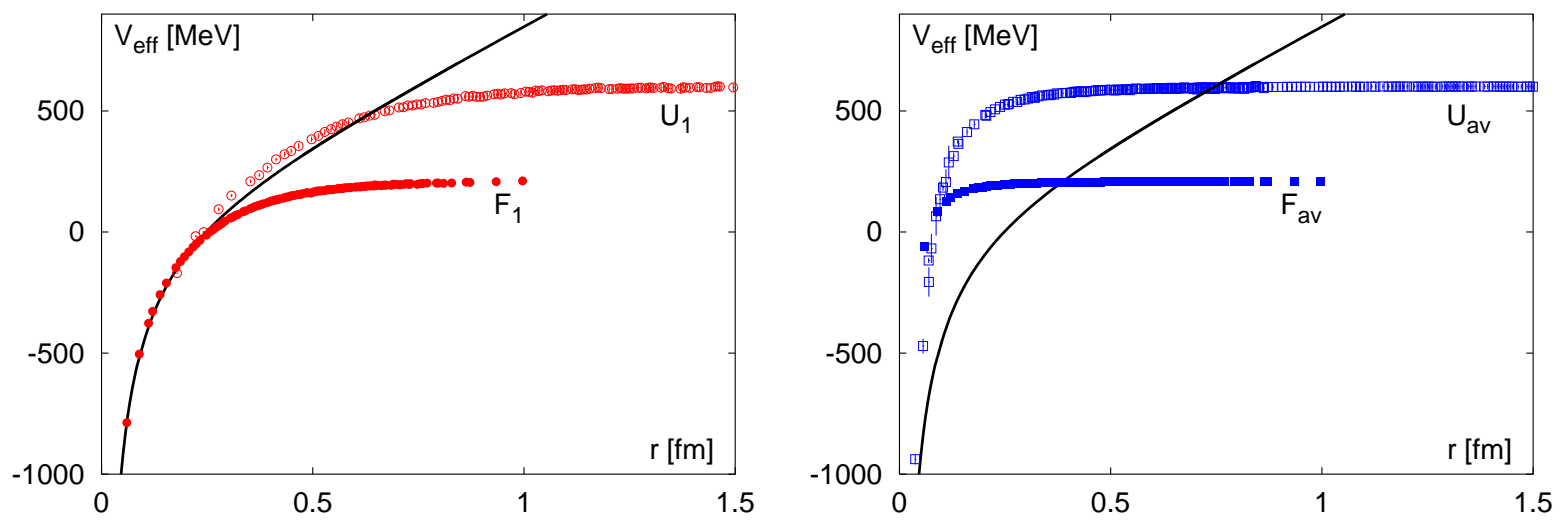

Fig. 3. Heavy quark free energies and energies in the color averaged and singlet channels at $T \simeq 1.4 T_{c}$. Results shown are obtained from calculations in pure $\mathrm{SU}(3)$ gauge theory [19].

responding non-relativistic Schrödinger equation,

$$
\left[2 m_{f}+\frac{1}{m_{f}} \nabla^{2}+V_{e f f}(r, T)\right] \Phi_{i}^{f}=E_{i}^{f}(T) \Phi_{i}^{f},
$$

where $m_{f}$ denotes the mass of the heavy quark flavor, e.g. $f \equiv$ charm or bottom, $i$ labels different quantum number channels and $E_{i}^{f}$ is the corresponding binding energy, i.e. the bound state mass is then given by $M_{i}^{f}=2 m_{f}+E_{i}^{f}$. Extensions of this approach taking into account relativistic corrections have recently been considered in 23 .

The "effective heavy quark potential", $V_{e f f}(r, T)$, models the influence of the medium on the $q \bar{q}$-system. This is then used to solve the bound state problem for a twobody system only. The main obstacle in such an approach clearly is to obtain a suitable ansatz for $V_{\text {eff }}(r, T)$. Here lattice calculations of the excess free energy of a pair of static quark anti-quark sources in a thermal medium, defined in Eqs. 1] and 2] have frequently been used to define an effective potential. The often used choice of the color averaged free energy as an effective potential, $V_{\text {eff }}(r, T) \equiv$ $F_{\text {av }}(r, T)$, may be questionable for two reasons. First of all Eq. 7 is meant to solve the bound state problem for a color singlet $q \bar{q}$-state. $F_{\mathrm{av}}(r, T)$, however, gives a weighted thermal average over $q \bar{q}$-states with arbitrary color orientation which can be understood in terms of, suitably defined, singlet and octet contributions. One thus might want to use the free energy for a $q \bar{q}$-pair fixed with relative color orientation corresponding to a color singlet state, $V_{e f f}(r, T) \equiv F_{1}(r, T)$. Moreover, it might be more suitable to use as an effective potential in Eq. [7 the energy, $U(r, T)$, rather then the free energy, $F(r, T)$, of two heavy quarks in a thermal bath. The former can be obtained from calculations of the free energy using the thermodynamic relation,

$$
U(r, T)=-T^{2} \frac{\partial F(r, T) / T}{\partial T},
$$

where $F$ and $U$ may correspond to the singlet or color averaged free energy and energy, respectively.
Depending on the ansatz used for the effective potential the solution of the Schrödinger equation, Eq. [7 will lead to different dissociation temperatures. The qualitative differences are obvious from Fig. 3] which shows for one value of the temperature, $T=1.4 T_{c}$, the color averaged free energy and energy of static quark anti-quark sources (left) as well as the corresponding quantities for the sources being fixed in a relative singlet orientation (right). As the free energy includes an additional entropy contribution, $F=U-T S$, potentials defined in terms of the energy are deeper than those defined in terms of free energies and thus will lead to higher dissociation temperatures. Likewise, the averaged (free) energies also receive contributions from octet configurations, which are repulsive at short distances. This leads to steeper (free) energies than in the singlet channel. Dissociation temperatures of heavy quark bound states determined from color averaged (free) energies are thus generically smaller than the corresponding values obtained with effective potentials defined in terms of singlet (free) energies. Some results for dissociation temperatures obtained by using as effective potential singlet free energies 21] and energies [22, respectively, are summarized in Tab. 1 Both calculations are based on lattice results obtained in the pure $\mathrm{SU}(3)$ gauge theory [19]. Similar results have also been obtained in 23 .

For temperatures above the transition temperature the free energies and energies that have been calculated now also for QCD with dynamical quarks [14,15] are quite similar to the pure gauge theory results. The dissociation temperatures displayed in Tab. 1 thus give a good description of the current status of potential model predictions. As may be obvious from Fig. 3 the largest dissociation temperatures are obtained from $U_{1}$.

Despite the model dependence entering through the choice of $V_{\text {eff }}$ some generic features are common to all the potential model calculations. Quite independently of the choice of $V_{\text {eff }}$ these calculations suggest that radial excitations in the charmonium system $\left(\chi_{c}, \psi^{\prime}\right)$ cannot exist as bound states in the high temperature phase. In fact, the disappearance of $\chi_{c}$ which will show up experimentally 


\begin{tabular}{|c||c|c|c||c|c|c|c|c|}
\hline state & $J / \psi$ & $\chi_{c}$ & $\psi^{\prime}$ & $\Upsilon$ & $\chi_{b}$ & $\Upsilon^{\prime}$ & $\chi_{b}^{\prime}$ & $\Upsilon^{\prime \prime}$ \\
\hline$E_{s}^{i}[\mathrm{GeV}]$ & 0.64 & 0.20 & 0.05 & 1.10 & 0.67 & 0.54 & 0.31 & 0.20 \\
\hline$T_{d} / T_{c}\left[\right.$ from $\left.F_{1}\right]$ & 1.1 & 0.74 & $0.1-0.2$ & 2.31 & 1.13 & 1.1 & 0.83 & 0.74 \\
$T_{d} / T_{c}\left[\right.$ from $\left.U_{1}\right]$ & $\sim 2.0$ & $\sim 1.1$ & $\sim 1.1$ & $\sim 4.5$ & $\sim 2.0$ & $\sim 2.0$ & - & - \\
\hline
\end{tabular}

Table 1. Dissociation temperatures in the charmonium and bottomonium system obtained by using pure gauge theory results for singlet free energies [21] (third row) and energies [22] (last row).

as a missing contribution to the $J / \psi$ yield seems to be a good probe for the transition temperature. Calculations based on using the singlet energy as an effective potential, furthermore, suggest that the ground states $\left(J / \psi, \eta_{c}\right)$ may still survive in the high temperature phase to temperatures as high as $2 T_{c}$. This, however, is in contrast to findings based on effective potentials constructed by using free energies rather than energies. This generically leads to smaller dissociation temperatures and, indeed, would suggest a dissociation of $J / \psi$ already at $T \simeq T_{c}$.

So far we have argued in this section in favor of using color singlet free energies (or energies) to discuss the fate of quarkonium bound states in the context of potential models. Nonetheless, one may also put forward arguments in favor of an effective potential which incorporates some of the color changing processes that take place in a thermal medium through the scattering of thermal gluons with the static sources. In fact, such processes have been discussed as a mechanism for direct quarkonium dissociation in a thermal medium 24. The absorption of thermal gluons can change the color orientation of the quark antiquark pair and put it into a color octet state. The analysis of color averaged (free) energies thus may have some justification also in the context of potential models.

\section{Heavy Quark Bound States from Spectral Analysis of Hadron Correlation Functions}

It should have become clear from the discussion in the previous section that an analysis of thermal properties of heavy quark bound states in terms of potential models and probably even the determination of singlet potentials from lattice calculations will require additional phenomenological consideration. On the other hand, as pointed out in the Introduction, an ab-initio approach to thermal hadron properties through lattice calculations exists and is based on the calculation of thermal hadron correlation functions,

$$
G_{H}(\tau, \boldsymbol{r}, T)=\left\langle J_{H}(\tau, \boldsymbol{r}) J_{H}^{\dagger}(0, \mathbf{0})\right\rangle,
$$

with $J_{H}=\bar{q}(\tau, \boldsymbol{r}) \Gamma_{H} q(\tau, \boldsymbol{r})$ denoting a hadron current where the projection onto appropriate quantum numbers, $H$, is controlled by a suitable product of gamma matrices $\Gamma_{H}$.

The influence of a thermal heat bath on hadron properties immediately becomes evident when one compares Euclidean time $(\tau)$ correlation functions of mesons in different quantum number channels. This is quite apparent for light quark mesons where thermal effects are related to the weakening of chiral symmetry breaking with increasing temperature and its restoration at high temperature. To illustrate the strong temperature effect on hadron correlation functions we thus briefly interrupt our discussion of heavy quark systems and have a look at the light quark, chiral sector of QCD. At low temperature chiral symmetry breaking and the breaking of the axial $U(1)$ symmetry lead to a splitting of hadronic states, which would be degenerate otherwise and would then also have identical correlation functions. In Fig. 4 we show pseudo-scalar $(\pi)$ and scalar $(\delta)$ meson correlation functions in a gluonic heat bath (quenched QCD) at temperatures below (left) and above (right) the transition temperature. The spectrum in the pseudo-scalar and scalar channels differs at low temperature due to the explicit breaking of the axial $U(1)$ symmetry which consequently leads to quite different correlation functions in Fig. 4(left). In fact, the difference will increase with decreasing quark mass values as the Goldstone nature of the pseudo-scalar than will lead to a flat correlation function. Above $T_{c}$ the almost perfect degeneracy of both correlation functions suggests that the axial $\mathrm{U}(1)$ symmetry is effectively restored. Moreover, the significant exponential drop of both correlation functions suggests that both modes are "quite heavy" in the deconfined phase of QCD (Fig. 4(right)).

The hadron correlation functions, $G_{H}(\tau, T)$, are directly related to spectral functions, $\sigma_{H}(\omega, T)$, which contain all the information on thermal modifications of the hadron spectrum in the quantum number channels, $H$,

$$
\begin{aligned}
& G_{H}(\tau, \boldsymbol{r}, T)= \\
& \quad \int_{0}^{\infty} \mathrm{d} \omega \frac{\mathrm{d}^{3} \boldsymbol{p}}{(2 \pi)^{3}} \sigma_{H}(\omega, \boldsymbol{p}, T) \mathrm{e}^{i \boldsymbol{p} \boldsymbol{r}} \frac{\cosh (\omega(\tau-1 / 2 T))}{\sinh (\omega / 2 T)}
\end{aligned}
$$

From the knowledge of e.g. the vector spectral function one obtains, for instance, direct information on thermal dilepton rates,

$$
\frac{\mathrm{d} W}{\mathrm{~d} \omega \mathrm{d}^{3} p}=\frac{5 \alpha^{2}}{27 \pi^{2}} \frac{\sigma_{V}(\omega, \boldsymbol{p}, T)}{\omega^{2}\left(\mathrm{e}^{\omega / T}-1\right)}
$$

Of course, it should be clear that the rates calculated in this way do not include any contributions arising from the feed-down of other quantum number channels into the vector channel [25].

Lattice studies of in-medium properties of hadrons have greatly advanced in their predictive power through the exploitation of the Maximum Entropy Method (MEM) [26 27. This allows the reconstruction of $\sigma_{H}(\omega, \boldsymbol{p}, T)$ at non-zero temperature for light as well as heavy quark 

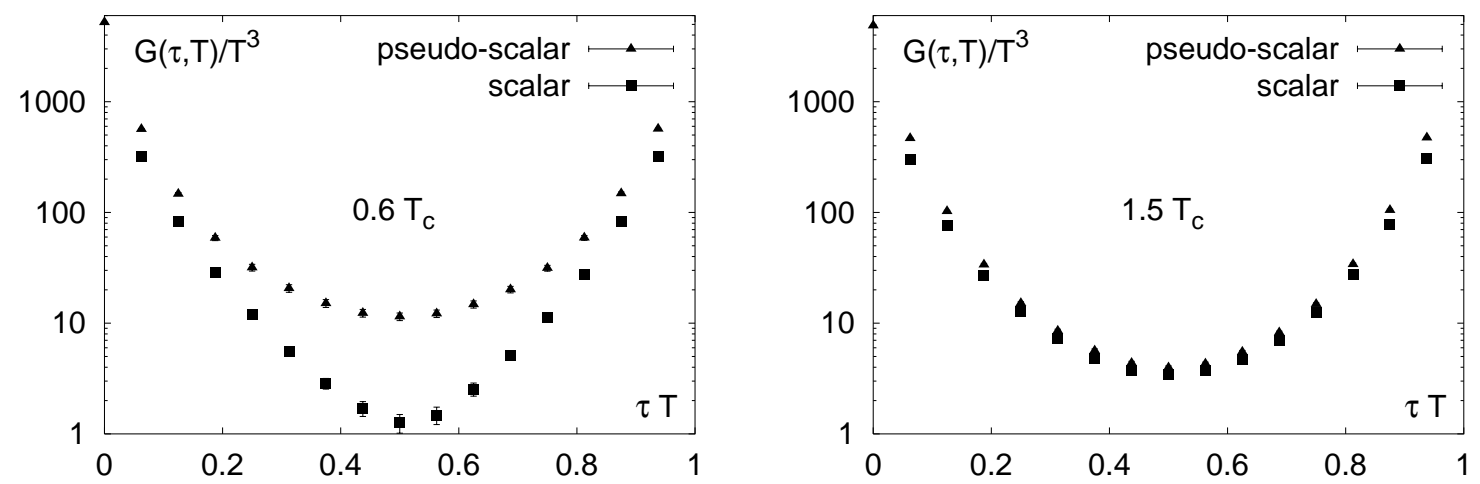

Fig. 4. Scalar $(\delta)$ and pseudo-scalar $(\pi)$ correlation functions at $T=0.6 T_{c}$ (left) and $T=1.5 T_{c}$ (right). Shown are results from calculations with light quarks on a $64^{3} \times 16$ lattice in quenched QCD.

bound states. We will concentrate in the following again on the heavy quark sector and, in particular, will discuss in how far the potential model calculations presented in the previous section are consistent with studies of charmonium spectral functions in the high temperature phase of QCD 28 29] 30]. We will restrict this discussion to the zero momentum sector of the charmonium system, $\sigma(\omega, T) \equiv$ $\sigma_{H}(\omega, \mathbf{0}, T)$, although also some preliminary results at nonzero momentum have been presented recently [31] and also some exploratory work for bottomonium has been performed 32.

As indicated in Eq. 10] $G_{H}(\tau, T) \sim \int \mathrm{d}^{3} \boldsymbol{r} G_{H}(\tau, \boldsymbol{r}, T)$ is related to the spectral functions $\sigma_{H}(\omega, T)$. However, an inversion of this integral equation, which would be needed to extract $\sigma_{H}(\omega, T)$ unambiguously, is generally not possible because lattice calculations only yield information on $G_{H}(\tau, T)$ at a finite, discrete set of Euclidean time steps, $\tau_{k} T=k / N_{\tau}$ with $k=0,1, \ldots N_{\tau}-1$, with $N_{\tau}$ denoting the temporal extent of the lattice. It is, however, possible to determine the most probable spectral function which describes the calculated data set $\left\{G_{H}\left(\tau_{k}, T\right) \mid k=\right.$ $\left.0, \ldots, N_{\tau}-1\right\}$ and respects known constraints on $\sigma_{H}(\omega, T)$ (positivity, asymptotic behavior, ...). This can be achieved using a Bayesian data analysis, e.g. the Maximum Entropy Method (MEM) 26. In the context of QCD calculations on the lattice this has been introduced in 27.

The MEM analysis has its own set of open problems. In particular, at finite temperature some ambiguities arise through the sensitivity of details of the spectral functions to the input spectral function used as default model to start the analysis 29 33. Moreover, also the high energy part of the spectral functions is strongly influenced by details of the scheme used to discretized the lattice fermion action (Wilson doublers 34 35]). This makes a "pointby-point" comparison of spectral functions determined by different groups difficult ${ }^{2}$.

A first feeling for the influence of a thermal heat bath on the structure of quarkonium correlation functions and

\footnotetext{
2 In any case, one should view the presently available set of calculations of spectral functions as exploratory leaving many possibilities for improvement.
}
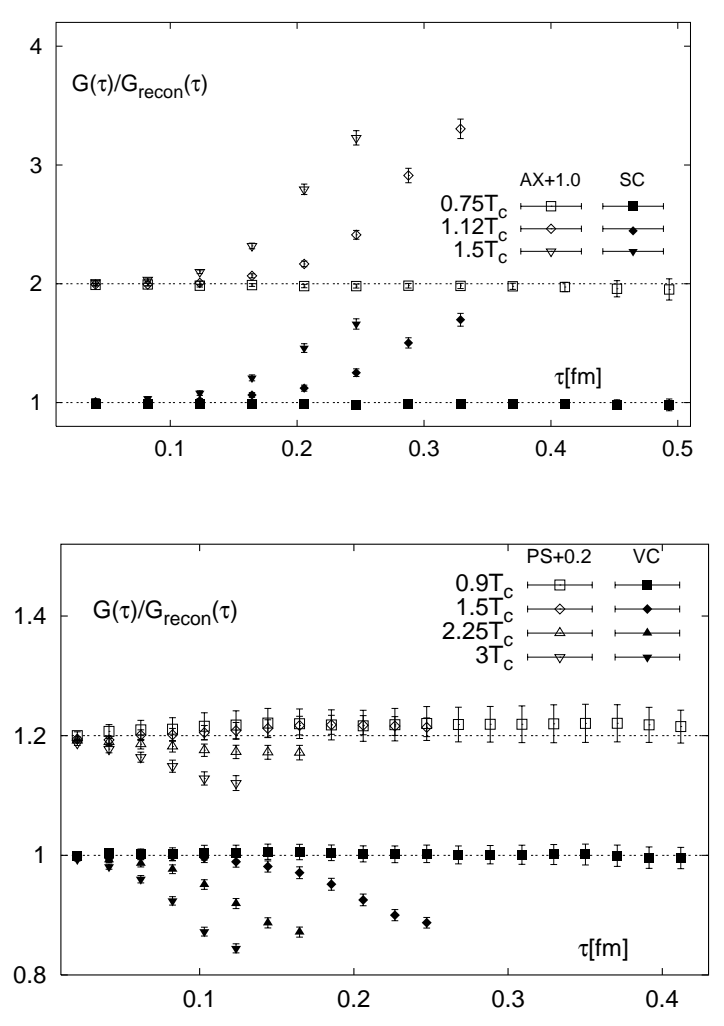

Fig. 5. Thermal correlation functions above $T_{c}$ in axial vector $\left(\mathrm{AX}: \chi_{c, 1}\right)$ scalar $\left(\mathrm{SC}: \chi_{c, 0}\right)$, pseudo-scalar (PS: $\left.\eta_{c}\right)$ and vector (VC: $J / \psi$ ) channels normalized to reconstructed correlation functions which are based on spectral functions calculated at $T^{*}=0.75 T_{c}$ (top) and $T^{*}=0.9 T_{c}$ (bottom) [29]. Note the different scales on the ordinates of both figures.

the size of medium modifications of a thermal spectral function can be obtained without relying entirely on the statistical MEM analysis by comparing directly the numerically calculated correlation functions at temperature $T$ with a correlation function, $G_{\text {recon }}(\tau, T)$, constructed 

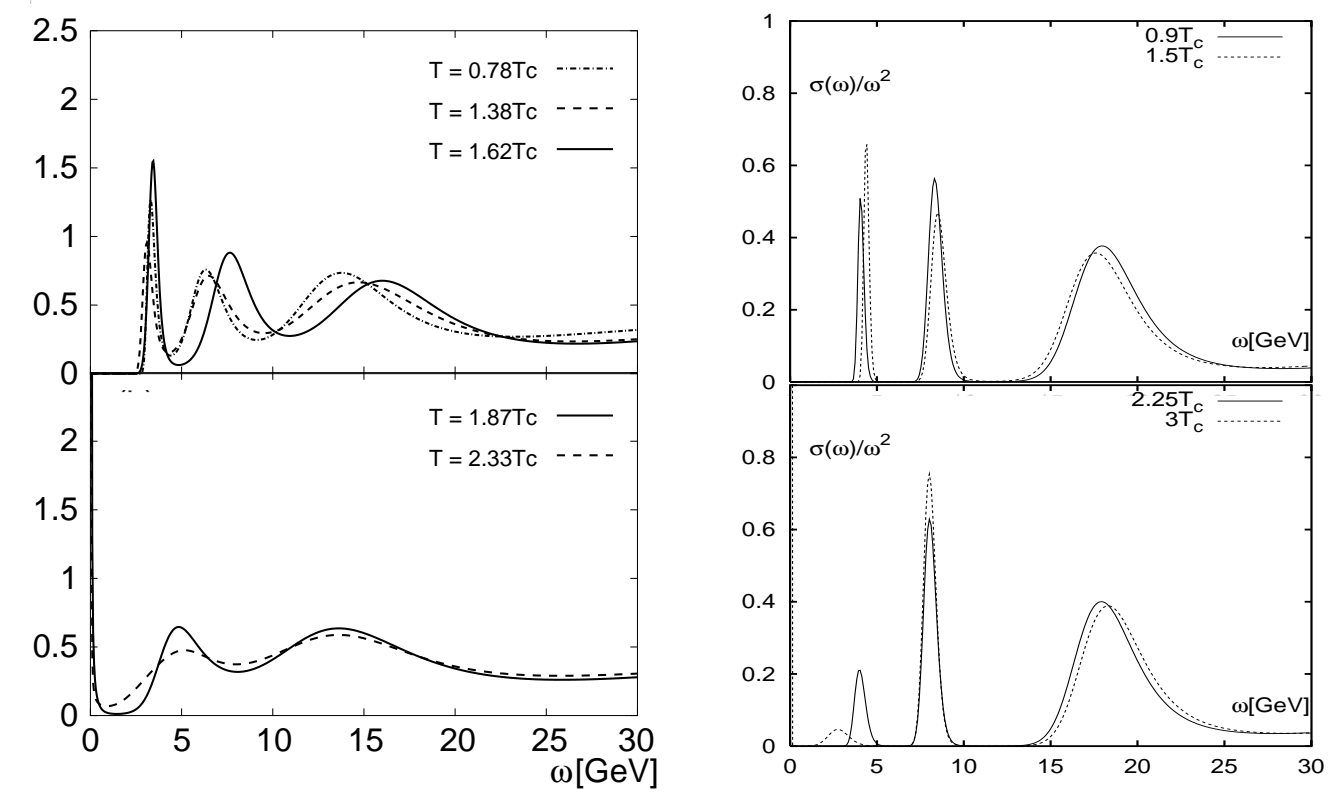

Fig. 6. Thermal vector spectral functions $(J / \psi)$ from MEM analyzes of meson correlation functions calculated in quenched QCD on anisotropic [28] (left) and isotropic [29] (right) lattices. The difference in scale on the ordinates of both figures is due to different normalizations used for the hadronic currents.

from the spectral function calculated at a smaller (zero) temperature, $T^{*}<T$, i.e.

$$
G_{\text {recon }}(\tau, T)=\int_{0}^{\infty} \mathrm{d} \omega \sigma_{H}\left(\omega, T^{*}\right) \frac{\cosh (\omega(\tau-1 / 2 T))}{\sinh (\omega / 2 T)} .
$$

In this way the trivial temperature dependence of the integration kernel is taken care of and any remaining discrepancies between the reconstructed correlation function and the actually calculated correlation function at temperature $T$ can be attributed to changes in the spectral function. Some results for charmonium correlation functions normalized in this way are shown in Fig. [5]

The ratio of correlation functions shown in Fig. [5]would equal unity, if the spectral functions would not depend on temperature. However, as can be seen the correlation functions for $P$-state charmonium (top), show strong thermal modifications already at temperatures slightly above $T_{c}$. On the other hand, the $S$-state correlators (bottom) show only little modifications up to $T \simeq 1.5 T_{c}$. In fact, no temperature dependence is visible in the pseudo-scalar channel $\left(\eta_{c}\right)$ up to $T=1.5 T_{c}$ and also modifications in the vector channel $(J / \psi)$ are only of the order of $10 \%$ at this temperature.

The reconstruction of charmonium spectral functions has been performed using technically different approaches, e.g. using point-like 28 29] or smeared [36 hadron sources on isotropic 29 or anisotropic 28,30 lattice with standard Wilson fermion 28 or improved Wilson fermion 29, 30] actions. The different calculations agree to the extent that no significant modification of $S$-state spectral functions is observed up to $1.5 T_{c}$, i.e. $J / \psi$ and $\eta_{c}$ survive as narrow bound states with unchanged mass up to this temperature. This is shown in the upper part of Fig. 6] for $J / \psi$. Current lattice calculations ${ }^{3}$ differ, however, on the structure of spectral functions for larger temperatures (lower part of Fig. 6). While it is concluded in 28 that the $J / \psi$ resonance disappears quite abruptly at $T \simeq 1.9 T_{c}$ the analysis of 29 suggests that the resonance disappears gradually; a resonance peak with reduced strength is still visible at $T=2.25 T_{c}$ and finally disappears completely at $T=3 T_{c}$.

For the radially excited states $\left(\chi_{c}\right)$ the strong temperature depends seen already on the level of the correlation functions (Fig. 5) also is apparent in the reconstructed spectral functions 29. No evidence for bound states is found in these channels above the transition temperature. Although finite temperature lattice calculations at present cannot resolve higher excited states in a given quantum number channel, e.g. the $\psi^{\prime}$ in the vector channel, it seems likely that also these excitations will get dissolved at $T_{c}$ or even earlier. All this is consistent with the revised potential model calculations in terms of a singlet free energy.

To get control over the detailed pattern of dissolution of the heavy quark resonances clearly requires more refined studies. It also should be noted that so far all existing lattice studies have been performed in the quenched approximation. Although virtual quark loops are not ex-

3 We stress again that current lattice calculations of spectral functions are influenced by lattice cut-off effects which show up most strongly at large energies, $\omega$. In fact, only the first, low energy peak in the spectral functions shown in Fig. [6] is physical and insensitive to changes of the lattice cut-off. The other two peaks have been shown to be lattice artifacts arising from "Wilson doublers" 34]. 


\begin{tabular}{llll}
\hline & SPS & RHIC & LHC \\
\hline$\sqrt{s}(\mathrm{GeV})$ & 18 & 200 & 5500 \\
$N_{c \bar{c}}$ & 0.2 & 10 & 200 \\
$N_{c h}$ & 1350 & 3250 & 16500 \\
\hline
\end{tabular}

Table 2. Estimate of the number of $c \bar{c}$-pairs created in central collisions at the SPS, RHIC and LHC [37. The estimate is based on production cross sections calculated in [39]. Also given is the total number of charged particles created in these collisions.

pected to modify the qualitative picture obtained from these calculations in the heavy quark sector of QCD one clearly has to understand their influence on a quantitative level. As discussed in the previous section also the analysis of free energies in pure gauge theories and QCD with dynamical quarks suggests that dynamical quarks do not alter the picture gained from quenched calculations significantly.

\section{Quarkonium suppression pattern in heavy ion collisions}

So far we have discussed possible modifications of quarkonium spectra in equilibrated quark-gluon matter kept at fixed temperature. Which impact the basic results of the spectral analysis of charmonium, i.e. (i) dissociation of excited states close to $T_{c}$, (ii) persistence of $J / \psi$ at least up to $1.5 T_{c}$, will have on observable charmonium yields in heavy ion collisions crucially depends on the fate of these states during the expansion of the hot and dense medium created in these collisions. To this extent it is important to understand what will happen to a quark anti-quark pair after its initial production in a hard collision during the subsequent expansion and cooling of the dense medium created around it. If the conditions in the medium are such that the initially created pair cannot form a bound state and will fly apart, it still may be the case that recombination processes play a significant role during the cooling of the hot medium [2 3]. If the latter is the case a scenario in which the observed charmonium yields may be interpreted in terms of a statistical hadronization model [4.5] seems likely to be valid. Contrary to the characteristic sequential suppression pattern [6 21] which would result from the standard screening scenario for quarkonium suppression [1] this would not lead to any threshold effects in the energy or temperature dependence of charmonium yields. In fact, it may well be that the latter scenario is favored for the kinematic conditions at SPS while the statistical hadronization picture could become valid at RHIC energies and even more so at the LHC. While at the SPS at best a single $c \bar{c}$-pair is created per collision quite a few pairs per collision can be created at RHIC and well above 100 pairs could be created in central collisions at the LHC. Such an estimate, taken from 37 38, is displayed in Tab. 2

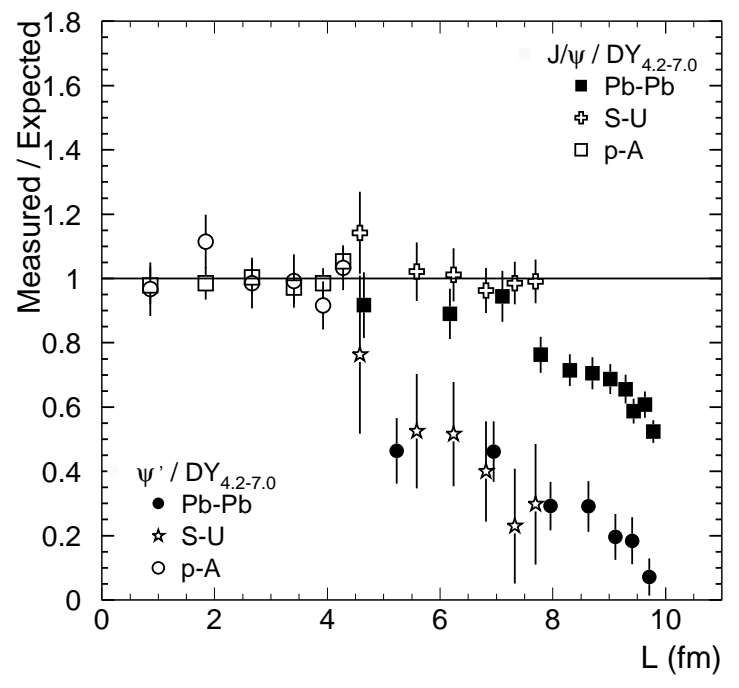

Fig. 7. Experimental information on charmonium suppression at the SPS: Shown is the ratio "measured value" /"expected value" for the relative yields $B_{\mu \mu} \sigma(J / \psi) / \sigma(D Y)$ and $B_{\mu \mu}^{\prime} \sigma\left(\psi^{\prime}\right) / \sigma(D Y)$ as a function of the nuclear absorption length $L$. The "expected value" takes into account the "normal" nuclear absorption of charmonium that traverses nuclear matter of thickness $L$.

As yields in recombination or hadronization models are proportional to $N_{c \bar{c}}^{2}$, charmonium yields grow much faster than the average charged particle multiplicities,

$$
N_{J / \psi} \sim N_{c \bar{c}}^{2} / N_{c h}
$$

At RHIC and LHC the charmonium yields thus could even increase rather than decrease irrespective of whether the original $c \bar{c}$-pair was dissolved by screening or other mechanisms.

If the formation of bound states is indeed controlled by the screening mechanism it may well be that the characteristic sequential suppression pattern can only be established at the SPS through a refined analysis of the charmonium system. The currently available data on $\psi^{\prime}$ and $J / \psi$ yields from the SPS 40.41, shown in Fig. 7 leave this possibility open. In fact, to substantiate the comparison of experimental results, which are commonly expressed in terms of the nuclear absorption length $L$ [41, with expectations based on the screening mechanism it will also be important to reach a better understanding of the relation between $L$, the energy density $\epsilon$ and the temperature $T$ of the dense matter created in a heavy ion collision. The relation between $L$ and $\epsilon$ is usually established through the Bjorken formula,

$$
\epsilon=\frac{\mathrm{d} E_{T} / \mathrm{d} \eta}{\tau A_{T}}
$$

where the geometrical overlap area $A_{T}$ is related to the impact parameter and can also be related to the absorption length $L$. Also the transverse energy per unit rapidity $\left(\mathrm{d} E_{T} / \mathrm{d} \eta\right)$, appearing in Eq. 114 is experimentally accessible. Some uncertainty, however, is introduced through the choice of the formation time $(\tau)$ used to characterize the 


\begin{tabular}{lll}
\hline$L[\mathrm{fm}]$ & $\epsilon\left[\mathrm{GeV} / \mathrm{fm}^{3}\right]$ & $T / T_{c}$ \\
\hline 4 & $\sim 1$ & $\sim 1.0$ \\
8 & $\sim 3$ & $\sim 1.16$ \\
10 & $\sim 4$ & $\sim 1.25$ \\
\hline
\end{tabular}

Table 3. Estimate of the relation between nuclear absorption length, energy density and temperature in units of the transition temperature for $\mathrm{Pb}-\mathrm{Pb}$ collisions at the SPS assuming $\epsilon_{c}=1 \mathrm{GeV} / \mathrm{fm}^{3}$.

initial state of the medium at the time where $c \bar{c}$ bound states might form. This generically is taken to be $1 \mathrm{fm}$. To further relate energy densities to thermal properties of the medium it is necessary to know the QCD equation of state which is taken from lattice calculations. In particular, the energy density at the transition point is presently known only with large errors, $\epsilon_{c}=(0.3-1.3) \mathrm{GeV} / \mathrm{fm}^{3}$.

Due to the rapid change of energy density and pressure in the transition region to the high temperature phase [42] small changes in the temperature correspond to large changes in the energy density or $L$. For orientation we list an estimate for the relation between these parameters in Tab. 3 which is taken from fiures presented in [1] and is based on an application of the Bjorken formula, Eq. 14] with $\tau=1 \mathrm{fm}$. The conversion of this energy density to a temperature scale has then been performed using the structure of the lattice equation of state by arbitrarily fixing the energy density at $T_{c}$ to be $1 \mathrm{GeV} / \mathrm{fm}^{3}$. This shows that the experimentally observed suppression pattern may actually correspond to a narrow temperature regime close to the transition temperature.

\section{Conclusions}

Quarkonium suppression has been observed in $\mathrm{Pb}-\mathrm{Pb}$ collisions at the SPS. The gross features are consistent with the original idea of $J / \psi$ suppression arising from screening of the heavy quark potential. It is conceivable that the suppression pattern observed at the SPS is specific to the kinematic conditions in these experiments and that the enhanced creation of $c \bar{c}$-pairs at RHIC and LHC may also lead to an enhancement of $J / \psi$ yields at these colliders.

Lattice calculations for charmonium spectral functions as well as refined potential model calculations suggest that the $J / \psi$ state can survive up to temperatures well above $T_{c}$ whereas excited states dissolve at or close to $T_{c}$. Given the current uncertainties in the conversion of experimental estimates for the initial energy density and theoretical relations between energy densities and temperatures the observed suppression pattern thus is in line with the assumption that it is caused by the dissolution of $\psi^{\prime}$ and $\chi_{c}$ states. At least on the theoretical side these uncertainties can and will be reduced through improvements in the determination of the transition temperature and the corresponding energy density.

\section{Acknowledgments}

This work has partly been supported by BMBF under grant No.06BI102 and funds of a Virtual Institute of the Helmholtz Association under grant No. VH-VI-041.

\section{References}

1. T. Matsui and H. Satz, Phys. Lett. B 178 (1986) 416.

2. R.L. Thews, M. Schroedter and J. Rafelski, Phys. Rev. C 63 (2001) 054905.

3. L. Grandchamp and R. Rapp, Phys. Lett. B 523 (2001) 60.

4. P. Braun-Munzinger and J. Stachel, Phys. Lett. B 490 (2000) 196.

5. L. Grandchamp and R. Rapp, Nucl. Phys. A 709 (2002) 415.

6. F. Karsch, M. T. Mehr and H. Satz, Z. Phys. C 37 (1988) 617.

7. F. Karsch and R. Petronzio, Phys. Lett. B 193 (1987) 105.

8. for an introductory discussion and further references see for instance: J. W. Harris and B. Müller, Ann. Rev. Nucl. Part. Sci. 46 (1996) 71; H. Satz, Rept. Prog. Phys. 63 (2000) 1511.

9. M. Bedjidian et al., Hard probes in heavy ion collisions at the LHC: Heavy flavour physics, (hep-ph/0311048), appeared in CERN yellow book, CERN-2004-009.

10. L. G. McLerran and B. Svetitsky, Phys. Rev. D 24 (1981) 450.

11. for an introduction to QCD thermodynamics on the lattice see for instance: F. Karsch, Lect. Notes Phys. 583 (2002) 209.

12. O. Philipsen, Phys. Lett. B 535 (2002) 138.

13. O. Kaczmarek, F. Karsch, F. Zantow and P. Petreczky, Phys. Rev. D 70 (2004) 074505.

14. O. Kaczmarek and F. Zantow, in preparation.

15. P. Petreczky and K. Petrov, Phys. Rev. D 70 (2004) 054503.

16. S. Necco and R. Sommer, Phys. Lett. B 523 (2001) 135 and Nucl. Phys. B622 (2002) 328.

17. M. Peter, Nucl. Phys. B501 (1997) 471; Y. Schröder, Phys. Lett. B447 (1999) 321.

18. O. Kaczmarek and F. Zantow, these proceedings, hep-lat/0502012

19. O. Kaczmarek, F. Karsch, P. Petreczky and F. Zantow, Phys. Lett. B 543 (2002) 41 and Nucl. Phys. Proc. Suppl. 129 (2004) 560.

20. S. Jacobs, M. G. Olsson and C. Suchyta III, Phys. Rev. D 33 (1986) 3338.

21. S. Digal, P. Petreczky and H. Satz, Phys. Lett. B 514 (2001) 57.

22. C.-Y. Wong, Heavy quarkonia in quark gluon plasma, hep-ph/0408020

23. G. E. Brown, C. H. Lee, M. Rho and E. Shuryak, Nucl. Phys. A 740 (2004) 171; E. V. Shuryak and I. Zahed, Phys. Rev. D 70 (2004) 054507.

24. D. Kharzeev and H. Satz, Phys. Lett. B 334 (1994) 155.

25. S. Digal, P. Petreczky and H. Satz, Phys. Rev. D 64 (2001) 094015.

26. R. K. Bryan, Eur. Biophys. J. 18 (1990) 165.

27. Y. Nakahara, M. Asakawa and T. Hatsuda, Phys. Rev. D 60 (1999) 091503.

28. M. Asakawa and T. Hatsuda Phys. Rev. Lett. 92 (2004) 012001. 
29. S. Datta, F. Karsch, P. Petreczky and I. Wetzorke, Phys. Rev. D 69 (2004) 094507

30. T. Umeda, R. Katayama, O. Miyamura and H. Matsufuru, Int. J. Mod. Phys. A 16 (2001) 2215.

31. S. Datta, F. Karsch, S. Wissel, P. Petreczky and I. Wetzorke, hep-lat/0409147

32. K. Petrov, these proceedings.

33. M. Asakawa, T. Hatsuda and Y. Nakahara, Prog. Part. Nucl. Phys. 46 (2001) 459.

34. T. Yamazaki et al. (CP-PACS Collaboration) Phys. Rev. D 65 (2002) 014501.

35. F. Karsch, E. Laermann, P. Petreczky and S. Stickan, Phys. Rev. D 68 (2003) 014504.

36. T. Umeda, K. Nomura and H. Matsufuru, hep-lat/0211003 3 .

37. R. L. Thews, hep-ph/0206179 in: Proceedings of Pan American Advanced Studies Institute on New States of Matter in Hadronic Interactions (PASI 2002), (Edts. H.-T. Elze, E. Ferreira, T. Kodama, J. Letesier, J. Rafelski und R. L. Thews), AIP Vol 631.

38. R. L. Thews, hep-ph/0302050 in: Hard Probes in Heavy Ion Collisions at the LHC (Edts. M. Mangano, H. Satz and U. Wiedemann), CERN-2004-009.

39. R. V. Gavai, S. Gupta, P. L. McGaughey, E. Quack, P. V. Ruuskanen, R. Vogt and X. N. Wang, Int. J. Mod. Phys. A 10 (1995) 2999.

40. M. Sitta [NA50 Collaboration], J. Phys. G 30 (2004) S1175.

41. B. Alessandro et al. [NA50 Collaboration], Eur. Phys. J. C 39 (2005) 335.

42. F. Karsch, E. Laermann and A. Peikert, Phys. Lett. B 478 (2000) 447. 
